Article

\title{
Invariant Means, Complementary Averages of Means, and a Characterization of the Beta-Type Means
}

\author{
Janusz Matkowski ${ }^{1}$ (D) and Paweł Pasteczka ${ }^{2, *}$ \\ 1 Institute of Mathematics, University of Zielona Góra, Szafrana 4a, PL-65-516 Zielona Góra, Poland; \\ j.matkowski@wmie.uz.zgora.pl \\ 2 Institute of Mathematics, Pedagogical University of Krakow, Podchorążych 2, PL-30-084 Kraków, Poland \\ * Correspondence: pawel.pasteczka@up.krakow.pl
}

Received: 4 September 2020; Accepted: 5 October 2020; Published: 12 October 2020 updates

\begin{abstract}
We prove that whenever the selfmapping $\left(M_{1}, \ldots, M_{p}\right): I^{p} \rightarrow I^{p},\left(p \in \mathbb{N}\right.$ and $M_{i}$-s are $p$-variable means on the interval $I$ ) is invariant with respect to some continuous and strictly monotone mean $K: I^{p} \rightarrow I$ then for every nonempty subset $S \subseteq\{1, \ldots, p\}$ there exists a uniquely determined mean $K_{S}: I^{p} \rightarrow I$ such that the mean-type mapping $\left(N_{1}, \ldots, N_{p}\right): I^{p} \rightarrow I^{p}$ is $K$-invariant, where $N_{i}:=K_{S}$ for $i \in S$ and $N_{i}:=M_{i}$ otherwise. Moreover $\min \left(M_{i}: i \in S\right) \leq K_{S} \leq \max \left(M_{i}: i \in S\right)$. Later we use this result to: (1) construct a broad family of $K$-invariant mean-type mappings, (2) solve functional equations of invariant-type, and (3) characterize Beta-type means.
\end{abstract}

Keywords: invariant means; complementary averages of means; characterizations; beta-type means MSC: primary 26E60; 39B22; secondary 39B12

\section{Preliminaries}

In the whole paper $I \subset \mathbb{R}$ stands for an interval, $p \in \mathbb{N}, p>1$ is fixed, and $\mathbb{N}_{p}:=\{1, \ldots, p\}$.

A function $M: I^{p} \rightarrow I$ is called a mean on $I$ if

$$
\min \left(x_{1}, \ldots, x_{p}\right) \leq M\left(x_{1}, \ldots, x_{p}\right) \leq \max \left(x_{1}, \ldots, x_{p}\right), \quad x_{1}, \ldots, x_{p} \in I,
$$

or, briefly, if

$$
\min \mathbf{x} \leq M(\mathbf{x}) \leq \max \mathbf{x}, \quad \mathbf{x}=\left(x_{1}, \ldots, x_{p}\right) \in I^{p} .
$$

The mean $M$ is called strict, if for all nonconstant vectors $\mathbf{x}$ these inequalities are sharp; and symmetric, if $M\left(x_{\sigma(1)}, \ldots, x_{\sigma(p)}\right)=M\left(x_{1}, \ldots, x_{p}\right)$ for all $x_{1}, \ldots, x_{p} \in I$ and all permutations $\sigma$ of the set $\mathbb{N}_{p}$. Mean $M$ is monotone if it is increasing in each of its variables. It is important to emphasize that every strictly monotone mean is strict.

A mapping $\mathbf{M}: I^{p} \rightarrow I^{p}$ is referred to as mean-type if there exist some means $M_{i}: I^{p} \rightarrow I, i \in \mathbb{N}_{p}$, such that $\mathbf{M}=\left(M_{1}, \ldots, M_{p}\right)$. For a mean-type mapping $\mathbf{M}: I^{p} \rightarrow I^{p}$ we denote a projection onto the $i$-th coordinate by $[\mathbf{M}]_{i}: I^{p} \rightarrow I$. In this case we obviously have $[\mathbf{M}]_{i}=M_{i}$ for all $i \in \mathbb{N}_{p}$.

We say that a function $K: I^{p} \rightarrow \mathbb{R}$ is invariant with respect to $\mathbf{M}$ (briefly $\mathbf{M}$-invariant), if $K \circ \mathbf{M}=K$.

Theorem 1 (Invariance Principle, [1]). If $\mathbf{M}: I^{p} \rightarrow I^{p}, \mathbf{M}=\left(M_{1}, \ldots, M_{p}\right)$ is a continuous mean-type mapping such that

$$
\max \mathbf{M}(\mathbf{x})-\min \mathbf{M}(\mathbf{x})<\max (\mathbf{x})-\min (\mathbf{x}), \quad \mathbf{x} \in I^{p} \backslash \Delta\left(I^{p}\right),
$$


where $\Delta\left(I^{p}\right):=\left\{\mathbf{x}=\left(x_{1}, \ldots, x_{p}\right) \in I^{p}: x_{1}=\ldots=x_{p}\right\}$ then there is a unique $\mathbf{M}$-invariant mean $\mathrm{K}: I^{p} \rightarrow I$ and the sequence of iterates $\left(\mathbf{M}^{n}\right)_{n \in \mathbb{N}}$ of the mean-type mapping $\mathbf{M}$ converges to $\mathbf{K}:=(K, \ldots, K)$ pointwise on $I^{p}$.

\section{A Family of Complementary Means}

Let us recall the following result.

Remark 1 (Matkowski [2]). Assume that $K: I^{2} \rightarrow I$ is a symmetric mean which is continuous and monotone. Then

(i) for an arbitrary mean $M_{1}: I^{2} \rightarrow I$ there is a unique mean $M_{2}: I^{2} \rightarrow I$ such that $K$ is $\left(M_{1}, M_{2}\right)$-invariant,

$$
K \circ\left(M_{1}, M_{2}\right)=K ;
$$

$\mathcal{K}_{M_{1}}:=M_{2}$, is referred to as a $K$-complementary mean for $M_{1}$; and we have

$$
\mathcal{K}_{M_{1}}=M_{2} \Longleftrightarrow \mathcal{K}_{M_{2}}=M_{1} ;
$$

(ii) if $M_{1}, M_{2}: I^{2} \rightarrow I$ are means such that $K$ is $\left(M_{1}, M_{2}\right)$-invariant, then there exists a unique mean $M: I^{2} \rightarrow$ I such that

$$
\min \left(M_{1}, M_{2}\right) \leq M \leq \max \left(M_{1}, M_{2}\right)
$$

and

$$
K \circ(M, M)=K
$$

moreover

$$
M=K .
$$

In this case, $p \geq 3$ the counterpart of part (i) of Remark 1 is false which shows the following

Example 1. Let $I=\mathbb{R}$, and $K=A$ where $A\left(x_{1}, x_{2}, x_{3}\right)=\frac{x_{1}+x_{2}+x_{3}}{3}$. The functions $M_{1}\left(x_{1}, x_{2}, x_{3}\right)=\frac{x_{1}+x_{2}}{2}$, $M_{2}\left(x_{1}, x_{2}, x_{3}\right)=x_{2}$ are means in $\mathbb{R}$. Then it is easy to see that there is no mean $M_{3}$ such that

$$
A \circ\left(M_{1}, M_{2}, M_{3}\right)=A \text {, }
$$

but a partial counterpart of part (ii) holds true.

The main result of this section reads as follows.

Theorem 2. Let $M_{1}, \ldots, M_{p}: I^{p} \rightarrow I$ be means. Assume that $K: I^{p} \rightarrow I$ is a continuous and monotone mean which is invariant with respect to the mean type mapping $\mathbf{M}:=\left(M_{1}, \ldots, M_{p}\right)$.

Then for every nonempty subset $S \subseteq \mathbb{N}_{p}$ there exists a unique mean $K_{S}(\mathbf{M}): I^{p} \rightarrow I$ such that $K$ in $\mathbf{K}_{S}(\mathbf{M})$-invariant, where $\mathbf{K}_{S}(\mathbf{M}): I^{p} \rightarrow I^{p}$ is given by

$$
\left[\mathbf{K}_{S}(\mathbf{M})\right]_{i}:= \begin{cases}K_{S}(\mathbf{M}) & \text { for } i \in S \\ M_{i} & \text { for } i \in \mathbb{N}_{p} \backslash S .\end{cases}
$$

Moreover, $\min \left(M_{i}: i \in S\right) \leq K_{S}(\mathbf{M}) \leq \max \left(M_{i}: i \in S\right)$.

Proof. In the case $S=\mathbb{N}_{p}$ the $\mathbf{K}_{S}(\mathbf{M})$-invariance of $K$ implies $K_{S}(\mathbf{M})=K$ and the statement is obvious. From now on we assume that $S \neq \mathbb{N}_{p}$. 
Denote briefly $M_{\vee}:=\max \left\{M_{i}: i \in S\right\}$ and $M_{\wedge}:=\min \left\{M_{i}: i \in S\right\}$. Fix $\mathbf{x} \in I^{n}$ arbitrarily. Define a function $T: I \rightarrow I^{p}$ by

$$
[T(\alpha)]_{i}:= \begin{cases}M_{i}(\mathbf{x}) & i \in \mathbb{N}_{p} \backslash S, \\ \alpha & i \in S .\end{cases}
$$

and $f: I \rightarrow I$ by $f(\alpha):=K \circ T(\alpha)$. Then, as $K$ is continuous and strictly increasing, so is $f$. Therefore in view of the equality $K \circ \mathbf{M}(\mathbf{x})=K(\mathbf{x})$ we obtain $f\left(M_{\vee}(\mathbf{x})\right) \geq K(\mathbf{x})$ and $f\left(M_{\wedge}(\mathbf{x})\right) \leq K(\mathbf{x})$. Thus, there exists unique number $\alpha_{\mathbf{x}} \in\left[M_{\wedge}(\mathbf{x}), M_{\vee}(\mathbf{x})\right]$ such that $f\left(\alpha_{\mathbf{x}}\right)=K(\mathbf{x})$. Now, as $\mathbf{x} \in I^{n}$ was arbitrary we define $K_{S}(\mathbf{M})(\mathbf{x}):=\alpha_{\mathbf{x}}$.

Then we have

$$
K(\mathbf{x})=f\left(\alpha_{\mathbf{x}}\right)=f\left(K_{S}(\mathbf{M})(\mathbf{x})\right)=\left(K \circ T \circ\left(K_{S}(\mathbf{M})\right)\right)(\mathbf{x})=\left(K \circ\left(K_{S}(\mathbf{M})\right)\right)(\mathbf{x}),
$$

which shows that $K$ is $\mathbf{K}_{S}(\mathbf{M})$-invariant.

Now we need to show that $K_{S}(\mathbf{M})$ is uniquely determined. Assume that $K$ is $\mathbf{K}_{S}(\mathbf{M})$-invariant and $K_{S}(\mathbf{M})(\mathbf{x}) \neq \alpha_{\mathbf{x}}$ for some $\mathbf{x} \in I^{p}$. Then, as $f$ is a monomorphism we obtain

$$
K \circ\left(\mathbf{K}_{S}(\mathbf{M})\right)(\mathbf{x})=\left(K \circ T \circ\left(K_{S}(\mathbf{M})\right)\right)(\mathbf{x}) \neq f\left(\alpha_{\mathbf{x}}\right)=K(\mathbf{x})
$$

contradicting the $\mathbf{K}_{S}(\mathbf{M})$-invariance.

Let us underline that we do not assume that means $M_{1}, \ldots, M_{P}$ are continuous. This is relatively recent approach to invariant property which was studied by the authors in [3].

The intuition beyond this theorem is the following. Once we have a continuous and monotone mean $K$ such that $\mathbf{M}$ is $K$-invariant mean we can unite a subfamily $\left(M_{s}\right)_{s \in S}$ into a single mean (denoted by $K_{S}(\mathbf{M})$ ) to preserve the $K$-invariance. In view of Theorem 1 , such a mean is unique. In this connection we propose the following

Definition 1. Let $K: I^{p} \rightarrow I$ be a continuous and monotone mean which is invariant with respect to the mean type mapping $\mathbf{M}:=\left(M_{1}, \ldots, M_{p}\right)$.

For each set $S \subset \mathbb{N}_{p}$ :

(i) the mean $K_{S}(\mathbf{M})$ is called a K-complementary averaging of the means $\left\{M_{i}: i \in S\right\}$ with respect to the invariant mean-type mapping $\mathbf{M}=\left(M_{1}, \ldots, M_{p}\right)$;

(ii) the mean-type mapping $\mathbf{K}_{S}(\mathbf{M})$ given by (1) is called a K-complementary averaging of the mean-type mapping $\mathbf{M}=\left(M_{1}, \ldots, M_{p}\right)$ with respect to the means $\left\{M_{i}: i \in S\right\}$.

Moreover, the set

$$
\mathfrak{K}(K, \mathbf{M}):=\left\{\mathbf{K}_{S}(\mathbf{M}): S \subseteq \mathbb{N}_{p}, S \neq \varnothing\right\}
$$

is called the family of all K-complementary averaging of the mean-type mapping $\mathbf{M}=\left(M_{1}, \ldots, M_{p}\right)$.

We can now reapply this result to the complementary of the establish a K-complementary of $\mathbf{K}_{S}(\mathbf{M})$ for the set $\mathbb{N}_{p} \backslash S$. More precisely we obtain

Corollary 1. Under the assumptions of Theorem 2 there exists unique mean $K_{S}^{*}(\mathbf{M}): I^{p} \rightarrow I$ such that $K$ is $\mathbf{K}_{S}^{*}(\mathbf{M})$-invariant, where $\mathbf{K}_{S}^{*}(\mathbf{M}): I^{p} \rightarrow I^{p}$ is given by

$$
\left[\mathbf{K}_{S}^{*}(\mathbf{M})\right]_{i}:= \begin{cases}K_{S}(\mathbf{M}) & \text { for } i \in S, \\ K_{S}^{*}(\mathbf{M}) & \text { for } i \in \mathbb{N}_{p} \backslash S .\end{cases}
$$

Moreover, $\min \left(M_{i}: i \in \mathbb{N}_{p} \backslash S\right) \leq K_{S}^{*}(\mathbf{M}) \leq \max \left(M_{i}: i \in \mathbb{N}_{p} \backslash S\right)$. 
Let us underline that the value $K_{S}^{*}(\mathbf{M})$ does not depend on $\mathbf{M}$ explicitly. The whole system of dependences is illustrated in Figure 1.

Observe that, as the mean $K_{S}^{*}(\mathbf{M})$ is uniquely determined, we obtain

$$
\mathbf{K}_{S}^{*}(\mathbf{M}) \in \mathfrak{K}(K, \mathbf{M}) \Longleftrightarrow \text { all means }\left(M_{i}: i \in \mathbb{N}_{p} \backslash S\right) \text { are equal to each other. }
$$

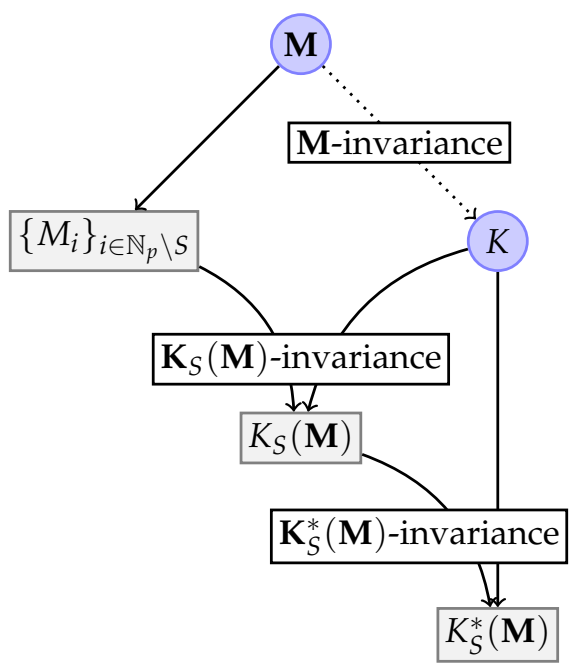

Figure 1. Map of dependencies between means. Rectangle vertexes are dependent on $S$. Dotted line means that there could exists more M-invariant mean satisfying the conditions of Theorem 2.

\section{Application in Solving Functional Equations}

Theorem 3. Let $\mathbf{M}=\left(M_{1}, \ldots, M_{p}\right)$ be a mean-type mapping such that $M_{1}, \ldots, M_{p}:(0, \infty)^{p} \rightarrow(0, \infty)$ are strictly monotonic and homogeneous. Then

(i) the sequence $\left(\mathbf{M}^{n}: n \in \mathbb{N}\right)$ of iterates of $\mathbf{M}$ converge uniformly on compact subsets to a mean-type mapping $\mathbf{K}=(K, \ldots, K)$, where $K$ is a unique $\mathbf{M}$-invariant mean.

(ii) $K$ is monotone, homogeneous and for every $S \subset \mathbb{N}_{p}$ the iterates of $\mathbf{K}_{S}(\mathbf{M})$ converge uniformly on compact subsets to a mean-type mapping $\mathbf{K}=(K, \ldots, K)$;

(iii) a function $F:(0, \infty)^{p} \rightarrow \mathbb{R}$ is continuous on the diagonal $\Delta\left((0, \infty)^{p}\right):=\left\{\left(x_{1}, \ldots, x_{p}\right) \in(0, \infty)^{p}: x_{1}=\cdots=x_{p}\right\}$ and satisfies the functional equation

$$
F \circ \mathbf{M}=F
$$

if and only if $F=\varphi \circ K$, where $\varphi:(0, \infty) \rightarrow \mathbb{R}$ is an arbitrary continuous function of a single variable;

(iv) a function $F:(0, \infty)^{p} \rightarrow \mathbb{R}$ is continuous on the diagonal $\Delta\left((0, \infty)^{p}\right)$ and satisfies the simultaneous system of functional equations

$$
F \circ \mathbf{K}_{S}(\mathbf{M})=F, \quad S \subset \mathbb{N}_{p} ;
$$

if and only if $F=\varphi \circ K$, where $\varphi:(0, \infty) \rightarrow \mathbb{R}$ is an arbitrary continuous function of a single variable (so (2) and (3) are equivalent)

Proof. The homogeneity and monotonicity of $M_{1}, \ldots, M_{p}$ imply their continuity (cf. ([4], Theorem 2)), so the invariance principle implies (i).

Now we prove that $K$ is monotone. Indeed, take two vectors $v, w \in(0, \infty)^{p}$ such that $v_{i} \leq w_{i}$ for all $i \in \mathbb{N}_{p}$ and $v_{i_{0}}<w_{i_{0}}$ for certain $i_{0} \in \mathbb{N}_{p}$. Then, as each $M_{i}$ is monotone, there exists a constant $\theta \in(0,1)$ such that $M_{i}(v) \leq \theta M_{i}(w)$ for all $i \in \mathbb{N}_{p}$. 
Then for all $n \in \mathbb{N}$ and $i \in \mathbb{N}_{p}$ we have

$$
\begin{aligned}
{\left[\mathbf{M}^{n}(v)\right]_{i} } & =\left[\mathbf{M}^{n-1}\left(M_{1}(v), \ldots, M_{p}(v)\right)\right]_{i} \leq\left[\mathbf{M}^{n-1}\left(\theta M_{1}(w), \ldots, \theta M_{p}(w)\right)\right]_{i} \\
& =\theta\left[\mathbf{M}^{n-1}\left(M_{1}(w), \ldots, M_{p}(w)\right)\right]_{i}=\theta\left[\mathbf{M}^{n}(w)\right]_{i} .
\end{aligned}
$$

In a limit case as $n \rightarrow \infty$ in view of the first part of this statement we obtain $K(v) \leq \theta K(w)<K(w)$. Thus, $K$ is monotone, which is (ii).

(iii) Assume first that $F:(0, \infty)^{p} \rightarrow \mathbb{R}$ that is continuous on the diagonal $\Delta\left((0, \infty)^{p}\right)$ and satisfies Equation (2). Hence, by induction,

$$
F=F \circ \mathbf{M}^{n}, \quad n \in \mathbb{N},
$$

By (ii) the sequence $\left(\mathbf{M}^{n}: n \in \mathbb{N}\right)$ converges to $\mathbf{K}=(K, \ldots, K)$. Since $F$ is continuous on $\Delta\left((0, \infty)^{p}\right)$, we hence get for all $\mathbf{x} \in(0, \infty)^{p}$,

$$
\begin{aligned}
F(\mathbf{x}) & =\lim _{n \rightarrow \infty} F\left(\mathbf{M}^{n}(\mathbf{x})\right)=F\left(\lim _{n \rightarrow \infty} \mathbf{M}^{n}(\mathbf{x})\right)=F(\mathbf{K}(\mathbf{x})) \\
& =F(K(\mathbf{x}), \ldots, K(\mathbf{x})) .
\end{aligned}
$$

Setting

$$
\varphi(t):=F(t, \ldots, t), \quad t \in(0, \infty),
$$

we hence get $F(\mathbf{x})=\varphi(K(\mathbf{x}))$ for all $\mathbf{x} \in(0, \infty)^{p}$.

To prove the converse implication, take an arbitrary function $\varphi: I \rightarrow \mathbb{R}$ and put $F:=\varphi \circ K$. Then, for all $\mathbf{x} \in(0, \infty)^{p}$, making use of the $K$-invariance with respect to $\mathbf{M}$, we have

$$
F(\mathbf{M}(\mathbf{x}))=(\varphi \circ K)(\mathbf{M}(\mathbf{x}))=\varphi(K(\mathbf{M}(\mathbf{x})))=\varphi(K(\mathbf{x}))=F(\mathbf{x}),
$$

which completes the proof of (iii).

(iv) we omit similar argument.

Part (ii) of this result gives rise to the following extension.

\subsection{General Complementary Process}

Once we have a mean-type $\mathbf{M}: I^{p} \rightarrow I^{p}$ and a continuous and monotone mean $K: I^{p} \rightarrow I$ which is $\mathbf{M}$-invariant let $\mathfrak{K}^{+}(\mathbf{M}, K)$ be the smallest family of mean-type mappings containing $\mathbf{M}$ which is closed under $K$-complementary averaging.

More precisely, for every $\mathbf{X} \in \mathfrak{K}^{+}(\mathbf{M}, K)$ and nonempty subset $S \subseteq \mathbb{N}_{p}$ we have $\mathbf{K}_{S}(\mathbf{X}) \in \mathfrak{K}^{+}(\mathbf{M}, K)$, too. We also define a family of means

$$
\mathfrak{K}_{0}(\mathbf{M}, K):=\left\{[\mathbf{X}]_{i}: \mathbf{X} \in \mathfrak{K}^{+}(\mathbf{M}, K) \text { and } i \in \mathbb{N}_{p}\right\}
$$

Obviously using notions from Theorem 2 and Corollary 1 we have

$$
\mathfrak{K}^{+}(\mathbf{M}, K) \supseteq \mathfrak{K}^{+}\left(\mathbf{K}_{S}(\mathbf{M}), K\right) \supseteq \mathfrak{K}^{+}\left(\mathbf{K}_{S}^{*}(\mathbf{M}), K\right)
$$

Furthermore, we have the following.

Proposition 1. Given an interval $I \subset \mathbb{R}, p \in \mathbb{N}$, and a mean-type mapping $\mathbf{M}:=\left(M_{1}, \ldots, M_{p}\right): I^{p} \rightarrow I^{p}$ which is invariant with respect to some continuous and monotone mean $K: I^{p} \rightarrow I$. Then

$$
\min \left(M_{1}, \ldots, M_{p}\right) \leq X \leq \max \left(M_{1}, \ldots, M_{p}\right) \quad \text { for all } X \in \mathfrak{K}_{0}(\mathbf{M}, K) \text {. }
$$

Its inductive proof is obvious in view of Theorem 2 (moreover part).

Now we prove that complementary means preserve symmetry. 
Proposition 2. If a continuous and monotone mean $K: I^{p} \rightarrow I$ is invariant with respect to a mean-type mapping $\mathbf{M}:=\left(M_{1}, \ldots, M_{p}\right): I^{p} \rightarrow I^{p}$ such that all $M_{i}$-s are symmetric, then $K$ and all means in $\mathfrak{K}_{0}(\mathbf{M}, K)$ are symmetric.

Proof. Fix a nonconstant vector $x \in I^{p}$ and a permutation $\sigma$ of $\mathbb{N}_{p}$. First observe that $K(x)=K \circ \mathbf{M}(x)=K \circ \mathbf{M}(x \circ \sigma)=K(x \circ \sigma)$, which implies that $K$ is symmetric.

As the family $\mathfrak{K}_{0}(\mathbf{M}, K)$ is generating by complementing, we need to show that symmetry is preserved by a single complement. Therefore, it is sufficient to show that the mean $K_{S}(\mathbf{M})$ defined in Theorem 2 is symmetric. However, using the notions therein, we have

$$
K \circ\left(\mathbf{K}_{S}(\mathbf{M})\right)(x)=K(x)=K(x \circ \sigma)=K \circ\left(\mathbf{K}_{S}(\mathbf{M})\right)(x \circ \sigma)
$$

By monotonicity of $K$, if $K_{S}(\mathbf{M})(x)<K_{S}(\mathbf{M})(x \circ \sigma)$ we would have

$$
K \circ\left(\mathbf{K}_{S}(\mathbf{M})\right)(x)=K(x)<K \circ\left(\mathbf{K}_{S}(\mathbf{M})\right)(x \circ \sigma)
$$

contradicting the above equality. Similarly we exclude the case $K_{S}(\mathbf{M})(x)>K_{S}(\mathbf{M})(x \circ \sigma)$. Therefore $K_{S}(\mathbf{M})(x)=K_{S}(\mathbf{M})(x \circ \sigma)$ which, as $x$ and $\sigma$ were taken arbitrarily, yields the symmetry of $K_{S}(\mathbf{M})$.

\section{An Applications to Beta-Type Means}

Following [5], for a given $k \in \mathbb{N}$ we define a $p$-variable Beta-type mean $\mathcal{B}_{p}: \mathbb{R}_{+}^{k} \rightarrow \mathbb{R}_{+}$by

$$
\mathcal{B}_{p}\left(x_{1}, \ldots, x_{p}\right):=\left(\frac{p x_{1} \cdots x_{p}}{x_{1}+\cdots+x_{p}}\right)^{\frac{1}{p-1}}
$$

This is a particular case of so-called biplanar-combinatoric means (Media biplana combinatoria) defined in Gini [6] and Gini-Zappa [7].

In order to formulate the next results, we adapt the notation that $A, G$ and $H$ are arithmetic, geometric and harmonic means of suitable dimension, respectively.

In [8], the invariance $G \circ(A, H)=G$, equivalent to the Pythagorean proportion, has been extended for arbitrary $p \geq 3$. In case $p=3$ it takes the form $G \circ(A, F, H)=G$, where

$$
F\left(x_{1}, x_{2}, x_{3}\right)=: \frac{x_{2} x_{3}+x_{3} x_{1}+x_{1} x_{2}}{x_{1}+x_{2}+x_{3}}, \quad x_{1}, x_{2}, x_{3}>0,
$$

and $H \leq F \leq A$. Hence, making use of Corollary 1 with $p=3, K=G, S=\{1,2\}$ we obtain the following.

Remark 2. For all $x_{1}, x_{2}, x_{3}$, the following inequality holds

$$
H\left(x_{1}, x_{2}, x_{3}\right) \leq \mathcal{B}_{3}\left(x_{1}, x_{2}, x_{p}\right) \leq A\left(x_{1}, x_{2}, x_{3}\right)
$$

and the inequalities are sharp for nonconstant vectors $x=\left(x_{1}, x_{2}, x_{3}\right) \in(0, \infty)^{3}$.

Passing to the main part of this section, first observe the following easy-to-see lemma.

Lemma 1. Let $p \in \mathbb{N}, p \geq 2$. Then there exists exactly one mean $M: I^{p} \rightarrow I$ such that $G \circ(A, \underbrace{M, \ldots, M}_{(p-1) \text { times }})=G$. Furthermore $M=\mathcal{B}_{p}$.

Its simple proof which is a straightforward implication of Theorem 2 is omitted. Based on this lemma it is natural to define a mean-type mapping $\mathbf{B}: I^{p} \rightarrow I^{p}$ by $[\mathbf{B}]_{1}:=A$ and $[\mathbf{B}]_{i}:=\mathcal{B}_{p}$ for all 
$i \in\{2, \ldots, p\}$. Then we have $G \circ \mathbf{B}=G$, which implies that the geometric mean is the unique B-invariant mean.

We are now going to establish the set $\mathfrak{K}^{+}(\mathbf{B}, G)$. It is quite easy to observe that all means in $\mathfrak{K}_{0}(\mathbf{B}, G)$ are of the form $\mathcal{H}_{p, \alpha}: I^{p} \rightarrow I(\alpha \in \mathbb{R})$ given by

$$
\mathcal{H}_{p, \alpha}\left(x_{1}, \ldots, x_{p}\right):=\left(x_{1} \cdots x_{p}\right)^{\frac{1-\alpha}{p}}\left(\frac{x_{1}+\cdots+x_{p}}{p}\right)^{\alpha}
$$

including $\mathcal{B}_{p}=\mathcal{H}_{p,-\frac{1}{p-1}}$. In the next lemma we show some elementary properties of the family $\left(\mathcal{H}_{p, \alpha}\right)$.

Lemma 2. Let $p \in \mathbb{N}$. Then

1. $\mathcal{H}_{p, \alpha}$ is reflexive for all $\alpha \in \mathbb{R}$, that is $\mathcal{H}_{p, \alpha}(x, \ldots, x)=x$ for all $x \in \mathbb{R}_{+}$,

2. $\mathcal{H}_{p, \alpha}$ is continuous for all $\alpha \in \mathbb{R}$ (as a $p$-variable function),

3. $\mathcal{H}_{p, \alpha}$ is a strict mean for all $\alpha \in\left[-\frac{1}{p-1}, 1\right]$,

4. $\mathcal{H}_{p, \alpha}$ is a symmetric function for all $\alpha \in \mathbb{R}$, that is $\mathcal{H}_{p, \alpha}(x \circ \sigma)=\mathcal{H}_{p, \alpha}(x)$ for all $x \in \mathbb{R}_{+}^{p}$ and $a$ permutation $\sigma$ of $\mathbb{N}_{p}$,

5. $\mathcal{H}_{p, 1}$ and $\mathcal{H}_{p, 0}$ are $p$-variable arithmetic and geometric means, respectively,

6. $\mathcal{H}_{p, \alpha}$ is increasing with respect to $\alpha$, that is $\mathcal{H}_{p, \alpha}(x)<\mathcal{H}_{p, \beta}(x)$ for every nonconstant vector $x \in \mathbb{R}_{+}^{p}$ and $\alpha, \beta \in \mathbb{R}$ with $\alpha<\beta$.

Proof. By the definition of $\mathcal{H}_{p, \alpha}$ we can easily verify that (1), (2), (4) and (5) holds.

From now on fix a nonconstant vector $x=\left(x_{1}, \ldots, x_{p}\right) \in \mathbb{R}_{+}^{p}$. By (4) we may assume without loss of generality that $x_{1} \leq \cdots \leq x_{p}$. Denote briefly

$$
g:=\sqrt[p]{x_{1} \cdots x_{p}} \quad \text { and } \quad a:=\frac{x_{1}+\cdots+x_{p}}{p} .
$$

By Cauchy inequality we have $x_{1}<g<a<x_{p}$. Moreover, by the definition $\mathcal{H}_{p, \alpha}(x)=a^{\alpha} g^{1-\alpha}$. Thus, for all $\alpha<\beta$ we have

$$
\mathcal{H}_{p, \beta}(x)=a^{\beta} g^{1-\beta}=a^{\alpha} g^{1-\alpha}\left(\frac{a}{g}\right)^{\beta-\alpha}>a^{\alpha} g^{1-\alpha}=\mathcal{H}_{p, \alpha}(x),
$$

which completes the proof of (6). The only remaining part to be proved is (3). However, applying (6), it is sufficient to show that

$$
\mathcal{H}_{p, 1}(x)<\max (x)=x_{p} \quad \text { and } \quad \mathcal{H}_{p,-\frac{1}{p-1}}(x)>\min (x)=x_{1} .
$$

By (5) we immediately obtain $\mathcal{H}_{p, 1}(x)=a<x_{p}$. For the second part observe that

$$
\mathcal{H}_{p,-\frac{1}{p-1}}(x)=\sqrt[p-1]{\frac{g^{p}}{a}}=\sqrt[p-1]{\frac{x_{1} \ldots x_{p}}{a}}>\sqrt[p-1]{\frac{x_{1} \ldots x_{p}}{x_{p}}}=\sqrt[p-1]{x_{1} \ldots x_{p-1}} \geq x_{1}
$$

which completes the proof.

Now we generalize Lemma 1 to the following form

Lemma 3. Let $p \in \mathbb{N}, p \geq 2$ and $\alpha \in \mathbb{R}^{p}$. Then $G \circ\left(\mathcal{H}_{p, \alpha_{1}}, \ldots, \mathcal{H}_{p, \alpha_{p}}\right)=G$, if and only if $\alpha_{1}+\cdots+\alpha_{p}=0$.

Its proof is obvious in view of the identity $G \circ\left(\mathcal{H}_{p, \alpha_{1}}, \ldots, \mathcal{H}_{p, \alpha_{p}}\right)=\mathcal{H}_{p, \frac{1}{p}\left(\alpha_{1}+\cdots+\alpha_{p}\right)}$. Having this proved, let us show the next important result. 
Theorem 4. Let $p \geq 2$. Then

$$
\begin{aligned}
& \mathfrak{K}^{+}\left((\underbrace{\left(A, \mathcal{B}_{p}, \ldots, \mathcal{B}_{p}\right.}_{(p-1) \text { times }}), G\right) \\
& \quad \subseteq\left\{\left(\mathcal{H}_{p, \alpha_{1}}, \ldots, \mathcal{H}_{p, \alpha_{p}}\right) \mid \alpha_{1}, \ldots, \alpha_{p} \in \mathbb{Q} \cap\left[-\frac{1}{p-1}, 1\right] \text { and } \alpha_{1}+\cdots+\alpha_{p}=0\right\} .
\end{aligned}
$$

In particular

$$
\mathfrak{K}_{0}=\left(\left(A, \mathcal{B}_{p}, \ldots, \mathcal{B}_{p}\right), G\right) \subseteq\left\{\mathcal{H}_{p, \alpha} \mid \alpha \in \mathbb{Q} \cap\left[-\frac{1}{p-1}, 1\right]\right\} .
$$

Proof. First observe that in view of Lemma $1 G$ is $\mathbf{B}$-invariant, and thus the set $\mathfrak{K}^{+}(\mathbf{B}, G)$ is well-defined. Now denote briefly

$$
\Lambda:=\left\{\left(\mathcal{H}_{p, \alpha_{1}}, \ldots, \mathcal{H}_{p, \alpha_{p}}\right) \mid \alpha_{1}, \ldots, \alpha_{p} \in \mathbb{Q} \cap\left[-\frac{1}{p-1}, 1\right] \text { and } \alpha_{1}+\cdots+\alpha_{p}=0\right\} .
$$

Obviously B $\in \Lambda$, so it is sufficient to prove that $\Lambda$ is closed with respect to $G$-complementary averaging. To this end take an arbitrary vector $\left(\alpha_{1}, \ldots, \alpha_{p}\right)$ real numbers such that $\mathbf{H}:=\left(\mathcal{H}_{p, \alpha_{1}}, \ldots, \mathcal{H}_{p, \alpha_{p}}\right) \in \Lambda$ and a nonempty subset $S \subset \mathbb{N}_{p}$.

By Theorem 2 there exists exactly one mean $G_{S}(\mathbf{H}): I^{p} \rightarrow I$ such that $G$ is $\mathbf{G}_{S}(\mathbf{H})$-invariant, where $\mathbf{G}_{S}(\mathbf{H}): I^{p} \rightarrow I^{p}$ is given by

$$
\left[\mathbf{G}_{S}(\mathbf{H})\right]_{i}:= \begin{cases}\mathcal{H}_{p, \alpha_{i}} & \text { for } i \in \mathbb{N}_{p} \backslash S, \\ G_{S}(\mathbf{H}) & \text { for } i \in S .\end{cases}
$$

On the other hand, in view of Lemma 3 we obtain that $G$ is invariant with respect to the mean-type mapping $\mathbf{H}_{0}: I^{p} \rightarrow I^{p}$ given by

$$
\left[\mathbf{H}_{0}\right]_{i}:=\left\{\begin{array}{ll}
\mathcal{H}_{p, \alpha_{i}} & \text { for } i \in \mathbb{N}_{p} \backslash S, \\
\mathcal{H}_{p, \beta} & \text { for } i \in S,
\end{array} \text { where } \beta=\frac{1}{|S|} \sum_{i \in S} \alpha_{i} .\right.
$$

As $G$ is both $\mathbf{G}_{S}(\mathbf{H})$-invariant and $\mathbf{H}_{0}$-invariant we obtain $G_{S}(\mathbf{H})=\mathcal{H}_{p, \beta}$, and consequently $\mathbf{G}_{S}(\mathbf{H})=\mathbf{H}_{0}$. Observe that $\mathbf{H}_{0} \in \Lambda$ is a straightforward implication of the equality

$$
\sum_{i \in \mathbb{N}_{p} \backslash S} \alpha_{i}+|S| \beta=\sum_{i \in \mathbb{N}_{p}} \alpha_{i}=0 .
$$

Now we show that $\beta \in \mathbb{Q} \cap\left[-\frac{1}{p-1}, 1\right]$, which would complete the proof. However, this is easy in view of the definition of $\beta$ and the analogous property $\alpha_{i} \in \mathbb{Q} \cap\left[-\frac{1}{p-1}, 1\right]$, which is valid for all $i \in S$.

Remark 3. Let us emphasize that inclusions in the above theorem are strict. More precisely, we can prove by simple induction that the denominator of $\alpha$ in irreducible form has no prime divisors greater than $p$.

Author Contributions: J.M. and P.P. contributed equally to the manuscript, and they read and approved the final manuscript. All authors have read and agreed to the published version of the manuscript.

Funding: This research received no external funding.

Conflicts of Interest: The authors declare no conflict of interest. 


\section{References}

1. Matkowski, J. Iterations of the mean-type mappings. In Iteration Theory (ECIT '08); Sharkovsky, A.N., Sushko, I.M., Eds.; Grazer Math. Ber., Bericht Nr 354; Institut für Mathematik, Karl-Franzens-Universität Graz: Graz, Austria, 2009; pp. 158-179.

2. Matkowski, J. Invariant and complementary quasi-arithmetic means. Aequ. Math. 1999, 57, 87-107. [CrossRef]

3. Matkowski, J.; Pasteczka, P. Mean-type mappings and invariance principle. arXiv 2020, arXiv:2005.10623.

4. Matkowski, J. Iterations of the mean-type mappings and uniqueness of invariant means. Ann. Univ. Sci. Budapest. Sect. Comput. 2013, 41, 145-158.

5. Himmel, M.; Matkowski, J. Beta-type means. J. Differ. Equ. Appl. 2018, 24, 753-772. [CrossRef]

6. Gini, C. Di una formula comprensiva delle medie. Metron 1938, 13, 3-22.

7. Gini, C.; Zappa, G. Sulle proprieta delle medie potenziate e combinatorie. Metron 1938, 13, $21-31$.

8. Matkowski, J. Means, generalized harmony proportion and applications. In Colloquium Mathematicum; Instytut Matematyczny Polskiej Akademii Nauk: Warsaw, Poland, 2020; Volume 160, pp. 109-118.

(C) 2020 by the authors. Licensee MDPI, Basel, Switzerland. This article is an open access article distributed under the terms and conditions of the Creative Commons Attribution (CC BY) license (http:/ / creativecommons.org/licenses/by/4.0/). 